\title{
The Trends of Modern Popular Science
}

\author{
Shufang Liang 1 Lianrong Zhao 2 \\ ${ }^{1}$ Shufang Liang (China university of geosciences (Beijing), school of humanities and economic management, master \\ degree candidate) \\ ${ }^{2}$ Lianrong Zhao (China university of geosciences (Beijing), school of humanities and economic management, \\ professor)
}

\begin{abstract}
Abstact
This article first expound the connotation of popular science, presents a general definition of popular science and analyze the stages of the popular science's history. I think that the process is devided into the stage before the popular science, traditional phase and modern popular science stage. Then I contrast the modern mode and the traditional mode of the popular science and figure out that the major change of the modern popular science's mode is the Interactivity .At last, by comparing with the traditional popular science, I analyze the new trend of the development of the popular science.
\end{abstract}

Key words: Modern popular science; New trend; Interactivity

\section{Introduction}

The level of development of science and technology is an important indicator of comprehensive national power. Technological progress depends on the improvement of national scientific literacy and the popularization of the science is essential to the national scientific literacy. Our country puts a high value on the popular science. And popular science is of great importance in the development strategy. 《National medium and long-term Scientific and Technological Development Plan 》 (2006-2020)takes enhancing the whole nation science and culture quality, creating a social environment which is conducive to technological innovation as an important national policies and measures and put forward the relevant policies. Such as implement national scientific literacy action plan, strengthen the construction of national science popularization ability and so on. Following the improvement and development of our society, modern popular science presents the regularity and characteristics are not the same as before. From the analysis of these changes, we can understand the connotation of the popular science more thoroughly. This will also contributes to the popular science career.

\section{The background of the popular science}

\subsection{The connotation of the popular science}

Science has a variety of English expressions such as popular science, science popularization and so on. Popular science appears as early as the 19th century in the West. But in our country, it appears in the middle of the 20th century. At first, it is the short for the national association of science and technology popularization. Not until 1956, has it been the abbreviation of the popular science. The popular science generated with the science and technology and developed with the improvement of it and so do the connotation of the popular science. About the connotation of the popular science, 《The Law of The Popularization of Science and Technology of China》has an indirect expression: The law shall be applicable to the state and society to popularize the knowledge and technology of science, To advocate the scientific method, spread the scientific thought and promote the scientific spirit. We should take the way of the public is easy to understand, accept and attend. So, we usually give the popular science such a definition: A society activity which the subjects of the popular science take the way is easy to understand, accept and attend for the public to advocate the scientific method, spread the scientific thought and promote the scientific spirit through some routes.

\subsection{The course of the popular science}

There is also some controversy about the course, but almost all the scholars devide it into three stages and regard the characteristic of the popular science as the standard of the staging. Huajie Liu and Shijin Huang think that we should devide it into the traditional stage, public understanding of science and the communication 
of science. However, Muli Huang devide it into the ancient popular science stage, and the modern stages. Most people hold the idea that they are the stage before the popular science, traditional phase and modern popular science stage. The following will discuss the specific three stages of the last method.

The stage before the popular science is from the time of the modem revolution of science to the middle of the 19th century. During this time, the popular science is in the infancy. Scientists spread their theories and methods all by themselves by conducting some experiments. As we know, Magdeburg hemispheres test is a typical example.

The traditional phrase is between the middle of the 19 th century and the middle of the 20th century. This is the all-round development stage of the popular science. Professional scientific organization begins to appear and represented by the emerging of the BAAS. What's more, the form of popular science presents a diversified trend. The popular science lectures, books and films become the mainstream of science popularization. Such as 《From the earth to the moon》 and 《Journey to the Moon》.

The modern popular science stage is from the middle of the 20th century. popular science has entered the phase of independence and has an important influence on the human society. The modern popular science shows different characteristics from the traditional stage. Shiqiang Ji presents four characteristics of the modern popular science.1) modern popular science is anti-scientism.2) Modern popular science gets more attention and participation from governments and public institutions. 3) The relationship between the subject and the object is more complex. 4) The contents and methods of modern science are more varied.

\subsection{The pattern of the popular science}

The popular science has developed for hundreds of years. It is not only a huge career but also a practical activity. The model and the structure vary from the development of the popular science. The traditional popular science consists of subjects, contents, objects and patterns of the popular science. The subjects are scientists and those who are interested in popular science. The contents are the basic knowledge and technology of science mostly. The objects are common publics. The form contains popular science books, movies and broadcast, some states even begin to build the libraries and museums. The pattern of the traditional popular science can be prescribed as follows.

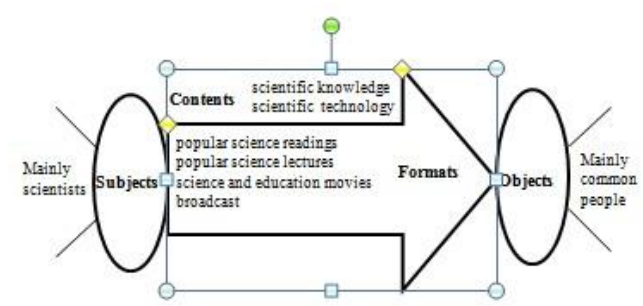

Fig. 1:the pattern of traditional popular science.

We can learn from the picture that the path of the traditional popular science is monodirectional, from the subjects to the objects directly. The objects are consumed to be those people who lack the scientific literacy and the contents are concerned to be right-on. That's why we can spread the scientific knowledge from the subjects to the objects directly. In this model, we ignore the importance of the objects' need and distinction. Eventually, this pattern is replaced by the modern popular science pattern.

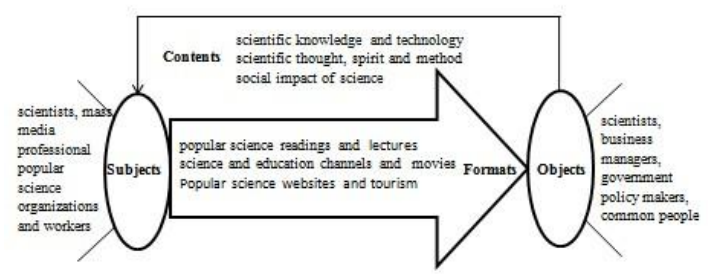

Fig. 2:the pattern of the modern popular science.

\section{The trends of the modern popular science}

Following with the rapid development of the science and technology, human society has been pushed forward. However, everything has two sides, the negative effects of the science and technology also revealed gradually. The popular science also reveals new characteristic and shows new tendency.

\subsection{The professionalism and diversification of the subject}

The public thinks that the scientist should undertake the major responsibility for science popularization for a long time. The scientists play an irreplaceable role in the popular science activity indeed. However, Along with the refine of social division of labor, many professional popular science group and organization appeared, such as mass media and professional popular science workers. This is actually a benign trend. On the 
one hand, the scientists can commit to the research. on the other hand, the running system of the popular science has been extended and improved.

\subsection{The nationality and subjectivity of the object}

With the rapid development of modern social material culture and the improvement of national science quality, the subjectivity of the public has been stimulated and the scope of the object has been expanded. Only a few people who have received a certain education have rigid demand of popular science in the traditional popular science. However, in the modern popular science, the object has been nationwide. There are mainly three categories. Firstly, general public is the maximum one. Including students, farmers, and working-class. Secondly, the scientists. This is a new group, scientists refers to those who are the expert in certain fields. They need the knowledge of other field for the growing permeability of different discipline. The last but not least, people who are engaged in the management especially the businessmen and the government community. The audiences of the popular science have the quality of self-conscious and creative. They have their own appeal about the contents and formats of the popular science. So, in the modern popular science, the appeals of the objects have been basis of the adjustment of the contents and formats.

\subsection{The extensibility of the contents}

The traditional popular science contents are mostly basic scientific knowledge and the basic phenomenon. However, the modern popular science contents are much more abundant and comprehensive. The contents include three sections. The first part is the basic scientific terms and conceptions. The second section is scientific processes and methods. The third part is the impact of the science and technology on the society, including the positive impact and the negative effect. In order to improve the scientific literacy of the whole nation, we should not only popularize the scientific technology and method, but also the scientific spirit and thought should be popularized.

\subsection{The innovation and timeless of the content}

Nowadays, the ways to spread are much more diverse than the past. A large number of novel approaches appear one by one, such as TV columns, websites and blogs. The instantaneity has been improved deeply. The objects can communicate with the subjects through various approaches. Furthermore, we combined the popular science activity with entertainment. Popular science tourism special line has been much popular in those years.

\subsection{Interaction in the process of popular science}

Compared with the traditional popular science, the direction of the modern popular science is bidirectional but not unidirectional. The objects of the popular science aren't passive to accept any more. The relationship between the object and the subject is equal and has two-way interaction relations. The feedbacks and appeals from the subject play a regulatory role to the direction and efficiency of the transmission of popular science. Huajie Liu ever put forward a represent a representative diagram. He holds the opinion that the system of the popular science transmission is a dynamic feedback system. The relationship between the subjects and the audiences is unidirectional and linear no longer but present an Interaction with each other in the network

\subsection{The Social relevance of the popular science}

The update rate of the modern popular science contents is faster and faster with the rapid development of the information technology. The traditional print media's state gradually decline in the modern popular science for the low update speed. However, the sudden events such as geologic hazard put forward high requirement of the timeless of the popular science activity. As Lijiao Xie's statistics, among the ten popular science events, more than half of them are closely related to public life. The melamine-contaminated dairy products event provoked the popular science boom about the food safety. Frequent geological disasters and the Worsening environmental pollution make the geological popular science to be bestowed favor of the popular science all over the world.

\section{Conclusion}

We have talked about the new trend above, but they are not the all. The Interaction in the process of the propagation of science and the timeless of the spread of popular science are also important new trend. The new trend put forward further requirement to us. The popular science career is facing new challenges. In order to develop the science popularization work of our country, we should recognize the development trend of modern science at first. We should innovate the forms, update 
the contents and pay more attention to the communication between the subject and the object to promote the progress of the popular science.

\section{References:}

[1]Huajie Liu. "Three Models and Three Stages of Science Communication,"[J] Proc. SCIENCE POPULARIZATION Theory and Practice, pp.10-18,2009(19).

[2]Shijin Huang. "The Subjectivity of the Audience in the Development of Science Communication," $[\mathrm{J}]$ Proc.The journal of east China university of science and Technology(Social Science Edition), pp.111-113+117,2007.

[3]Muli Huang. "The Changed Modes of Science Popularization in Internet Society,' $[\mathrm{J}]$ Proc. The journal of Guangxi university(philosophy and social science), pp.15-17,2002.

[4]Hongcang Zhou. "Exploration of the Public Promulgations' Educational Pattern,'[D] Proc. Jiangsu: Nanjing Normal University, 2006.

[5]Guohao Dong. "Discuss about the Two Peaks of Popular Science in the 20th Century in China,'[D] Proc. Sichuan: Chengdu Univerisity of Technology, 2008.

[6]Shiqiang Ji,Jianxia Ren. "Analysis of Characteristics of Modern Science," $[\mathrm{J}] \quad$ Proc. The journal of Ningbo radio and television university, pp.112-115,2006.

[7]Lijiao Xie. "The Formation of 'the Popular Science of Public Events'and the Analysis on Its Mechanism," [J]. Proc. SCIENCE POPULARIZATION, pp.32-36,2010. 